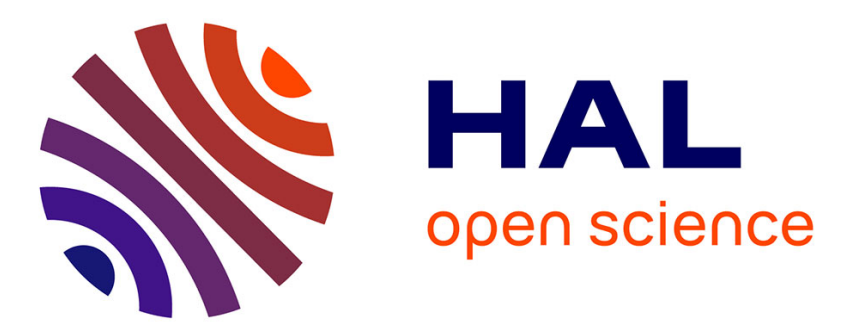

\title{
The fast dynamics of cavitation bubbles within water confined in elastic solids
}

Olivier Vincent, Philippe Marmottant, S. Roberto Gonzalez-Avila, Keita Ando, Claus-Dieter Ohl

\section{- To cite this version:}

Olivier Vincent, Philippe Marmottant, S. Roberto Gonzalez-Avila, Keita Ando, Claus-Dieter Ohl. The fast dynamics of cavitation bubbles within water confined in elastic solids. Soft Matter, 2014, 10 (10), pp.1455. 10.1039/c3sm52697f . hal-01082221

\section{HAL Id: hal-01082221 \\ https://hal.univ-grenoble-alpes.fr/hal-01082221}

Submitted on 14 Nov 2014

HAL is a multi-disciplinary open access archive for the deposit and dissemination of scientific research documents, whether they are published or not. The documents may come from teaching and research institutions in France or abroad, or from public or private research centers.
L'archive ouverte pluridisciplinaire HAL, est destinée au dépôt et à la diffusion de documents scientifiques de niveau recherche, publiés ou non, émanant des établissements d'enseignement et de recherche français ou étrangers, des laboratoires publics ou privés. 

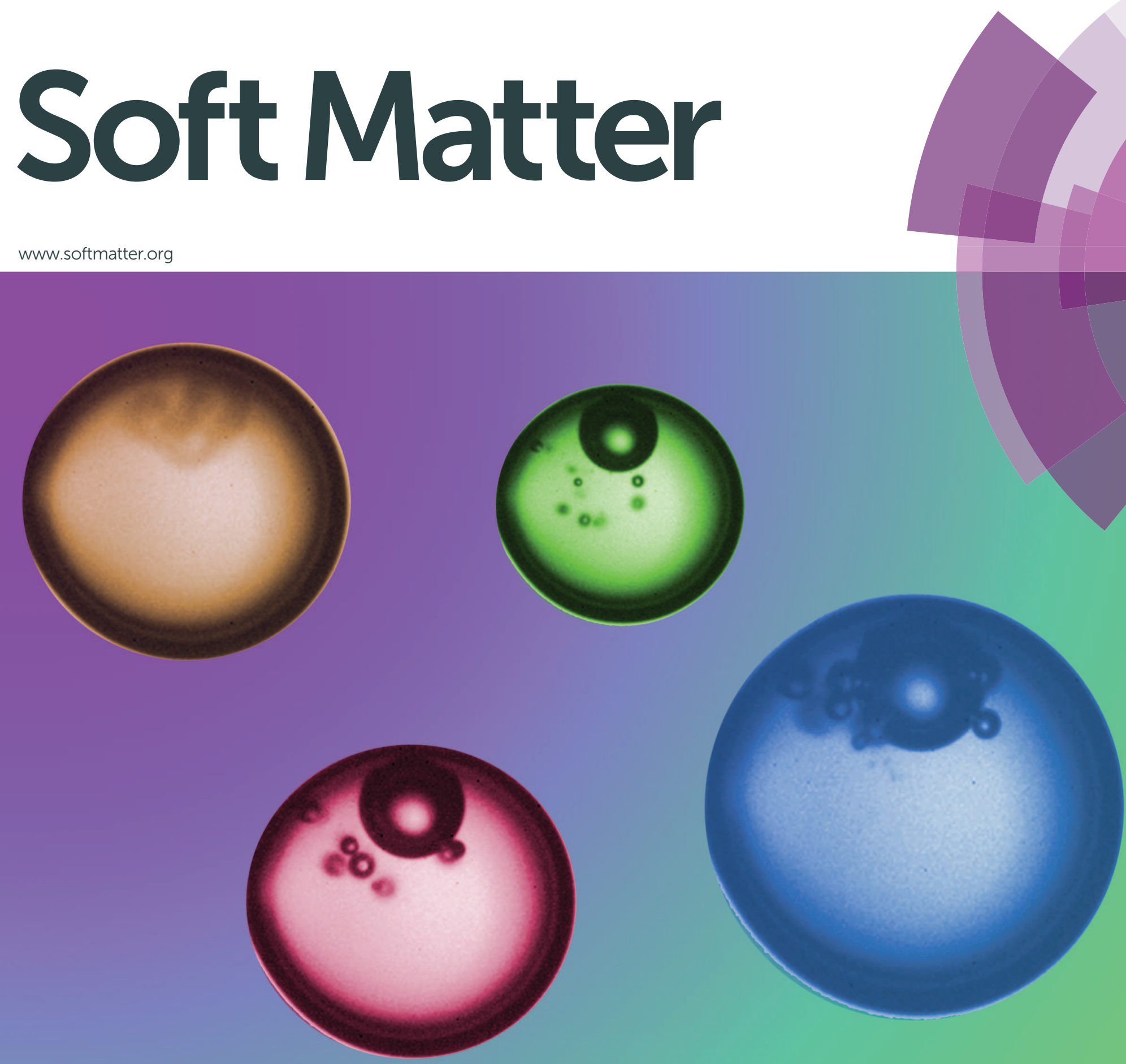

ISSN 1744-683X

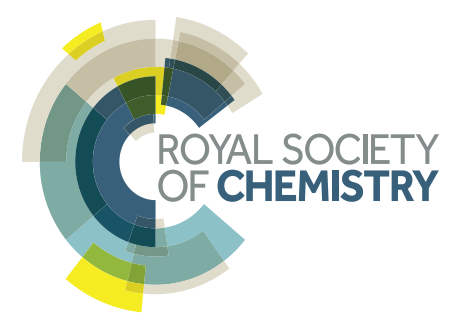

\section{PAPER}

Olivier Vincent, Philippe Marmottant et al.

The fast dynamics of cavitation bubbles within water confined in elastic solids 


\title{
The fast dynamics of cavitation bubbles within water confined in elastic solids
}

\author{
Olivier Vincent, $\dagger^{\star a}$ Philippe Marmottant, ${ }^{* a}$ S. Roberto Gonzalez-Avila,,$^{\mathrm{b}}$ Keita Ando $\S^{\mathrm{c}}$ \\ and Claus-Dieter $\mathrm{Ohl}^{\mathrm{b}}$
}

Many applications such as ultrasonic cleaning or sonochemistry use the ability of bubbles to oscillate and drive liquid flow. But bubbles have also received attention in porous media, where drying may cause cavitation, a phenomenon occurring in plant tissues. Here we explore the dynamics of cavitation bubbles when the liquid is fully entrapped in an elastic solid, using light scattering, laser strobe photography and high speed camera recordings. Our experiments show unexpectedly fast bubble oscillations in volume. They depend on the confinement size and elasticity, which we explain with a simple model where liquid compressibility is a key parameter. We also observe rich non-spherical dynamics, with ejection away from the walls and bubble fragmentation, which reveal extreme fluid motion at short timescales.

Received 22nd October 2013

Accepted 28th November 2013

DOI: $10.1039 / c 3 s m 52697 f$

www.rsc.org/softmatter and more generally during the drying of porous media. ${ }^{17,18}$ Unraveling bubble dynamics in these systems may allow, among other things, a better comprehension of acoustic emissions and embolism dynamics in trees, ${ }^{\mathbf{1 4 , 1 9}}$ and of the timescales of spore ejection in ferns, ${ }^{20}$ and more generally can provide insipiration for the design or monitoring of systems based on cavitation or metastable liquids.

Bubble volume oscillations are of particular interest. In a free liquid, they are described by Minnaert's theory, ${ }^{5}$ considering the bubbles as mass-spring systems where the mass arises from the inertia of the displaced liquid, and the stiffness comes from the compressibility of the gas inside the bubble. In this article, we show experimentally and theoretically that confinement not only introduces dramatic changes to this classical result of bubble dynamics, but also entails peculiar hydrodynamic phenomena which result from fast fluid motion.

\section{Cavitation and relaxation of tension}

To investigate confined bubble dynamics, we use water confined in spherical microcavities, of radius $R_{\mathrm{c}}$, inside a stiff polyhydroxyethylmethacrylate (pHEMA) hydrogel (Fig. 1a). This system, proposed recently, ${ }^{21}$ allows the generation of negative pressure (tension) in water by evaporation from the gel. We described the fabrication of these samples in a recent paper. ${ }^{22}$ Tension builds slowly as the gel dehydrates, and water experiences a stretched, metastable state. At liquid pressures down to about $-20 \mathrm{MPa}$, spontaneous cavitation in the metastable liquid results in the creation of a bubble (Fig. $1 \mathrm{~b}$ and c), relaxing the tension ${ }^{21,22}$ (Fig. 1b).

This sudden relaxation has two effects. First, the previously stretched liquid contracts due to water compressibility, leaving space for the cavitation bubble. Second, the inward force due to 
(a)

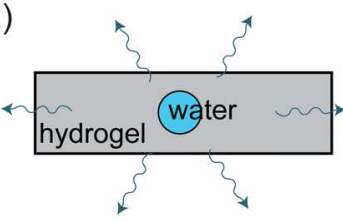

(b)

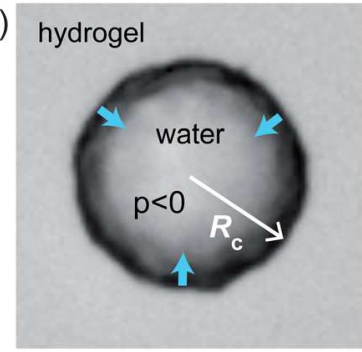

(d)

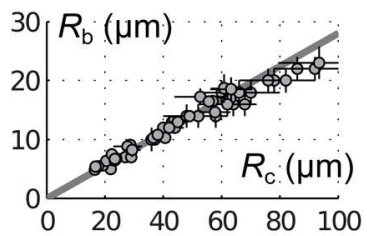

(c)

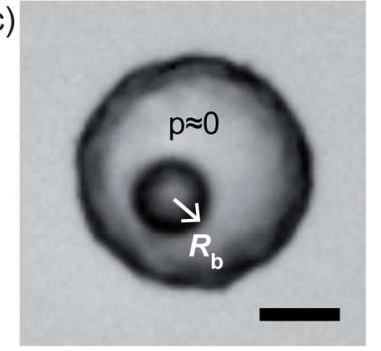

Fig. 1 (a) Sketch of a sample: a water microcavity is embedded near the middle plane of a hydrogel $(0.6 \mathrm{~mm}$ thick) through which water can flow and evaporate at the free surface (arrows) towards a subsaturated atmosphere, lowering water pressure in the cavity and making the liquid metastable. (b) Optical image of a microcavity before cavitation. Water is under negative pressure (tension) and pulls on the walls of the microcavity. (c) The microcavity after spontaneous cavitation. Tension has been relaxed by the expansion of the bubble. Scale bar:20 $\mu \mathrm{m}$. (d) Temporary equilibrium radius as a function of microcavity radius, showing a linear dependence. The solid line represents $R_{\mathrm{b}} / R_{\mathrm{C}}=0.28$

liquid tension, pulling the elastic solid towards the center of the cavity (Fig. 1b), disappears (Fig. 1c). This makes the microcavity expand, leaving some additional free space. The initial size of the bubble $R_{\mathrm{b}}$ is thus a combined consequence of these two effects.

Assuming the linear elasticity of both the liquid and the solid, one would expect $R_{\mathrm{b}}$ to be proportional to the microcavity size $R_{\mathrm{c}}$, which we verified experimentally by measuring the initial bubble size with high speed camera recordings of the cavitation process (Fig. 1d). We get $R_{\mathrm{b}}=\beta R_{\mathrm{c}}$, with $\beta \simeq 0.3$. Varying $R_{\mathrm{c}}$ thus provides a straightforward method to control $R_{\mathrm{b}}$ and test the dependence of the dynamics on the bubble size.

Note that after this initial bubble expansion, a second but slower expansion follows, due to poroelastic fluxes in the porous hydrogel surrounding the cavity, ${ }^{22}$ characterized by a diffusion coefficient $D \sim 10^{-11} \mathrm{~m}^{2} \mathrm{~s}^{-1}$. However, for timescales shorter compared to $\tau_{\text {poro }}=R_{\mathrm{c}}{ }^{2} / D$, which is on the order of seconds, these fluxes are negligible and the liquid is effectively trapped inside the microcavity.

In this paper we are interested in the bubble dynamics at short (sub-microsecond) timescales, where we can thus consider that there is a fixed quantity of water trapped in an elastic solid. We aim to characterize the vibratory dynamics of the cavitation bubble in this fully confined environment. To achieve this goal, several experimental setups were used and will be described when needed in the paper.

\section{Volume oscillations: experiments}

Volume oscillations are naturally excited as the bubble expands from $R=0$ to $R=R_{\mathrm{b}}$ due to the tensile forces of the liquid. To

track these oscillations, we developed a setup sketched in Fig. 2a, using a photodiode on which the optical image of the cavity is projected. As the bubble expands, more light is scattered and the light intensity detected by the photodiode decreases. Our recordings show a very reproducible pattern of typically 6 to 8 damped oscillations with a well-defined frequency $f$ in the $\mathrm{MHz}$ range (Fig. 2b). We characterized the dependence of this frequency on the cavity size (Fig. 2c), showing a clear dependence of the form

$$
f=\mathscr{C} / R_{\mathrm{c}}
$$

with $\mathscr{C}=120 \pm 10 \mathrm{~m} \mathrm{~s}^{-1}$, over more than a decade of values of $R_{\mathrm{c}}$. Using $R_{\mathrm{b}}=\beta R_{\mathrm{c}}$, the frequency is also inversely proportional to the bubble radius: $f=\mathscr{D} / R_{\mathrm{b}}$, with $\mathscr{D}=\beta \mathscr{C} \simeq 35 \mathrm{~m} \mathrm{~s}^{-1}$. This peculiar dependence is similar to the one predicted by Minnaert ${ }^{5}$ for the volume oscillations of air bubbles in bulk water $\left(f=\mathscr{D}_{\mathrm{M}} / R_{\mathrm{b}}\right.$, with $\left.\mathscr{D}_{\mathrm{M}} \simeq 3 \mathrm{~m} \mathrm{~s}^{-1}\right)$ and suggests that we are detecting such oscillations. However, the present oscillations are an order of magnitude faster and might thus be of different nature.

To clarify that point, we used a laser strobe photography setup (Fig. 3a), capable of imaging the bubble at a very short time $t$ after nucleation. For all time delays explored (up to $500 \mathrm{~ns}$ ) the bubble contour was circular (Fig. 3b), with a radius $R$ $(t)$ reported in Fig. 3c. Bubble dynamics cannot be directly inferred from the raw radius data (Fig. 3c, inset) as different microcavity sizes were used for each point, each giving rise to different dynamics according to the results from the photodiode setup. However, when the radius is scaled with the cavity size $R_{\mathrm{c}}$, and the time is scaled with the expected oscillation period $T_{\exp }=R_{\mathrm{c}} / \mathscr{C}$ (from eqn (1)), all data points finely collapse on the same curve, clearly showing an oscillation in the same (a)

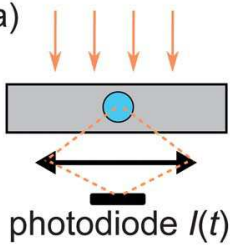

(b)

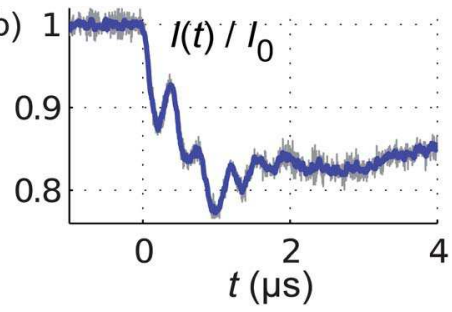

(c)

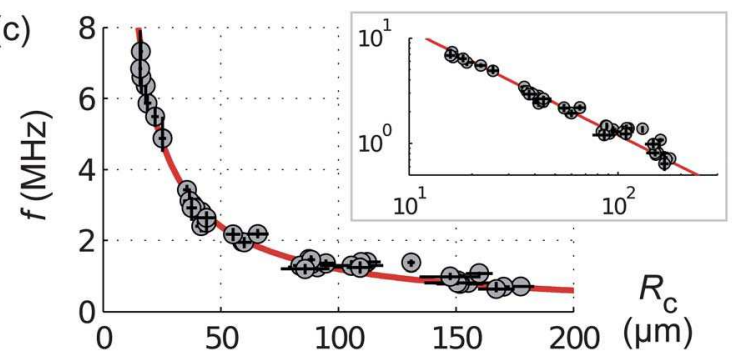

Fig. 2 Bubble dynamics measured by scattering (extinction). (a) Simple sketch of the experimental setup. (b) Typical signal, with cavitation occuring at $t=0$, showing bubble oscillation. (c) Frequency of bubble oscillation as a function of microcavity radius $R_{\mathrm{c}}$. The error bars are drawn in black, and the solid line represents $f \times R_{\mathrm{c}}=120 \mathrm{~m} \mathrm{~s}^{-1}$. Inset: log-log plot. 
(a)

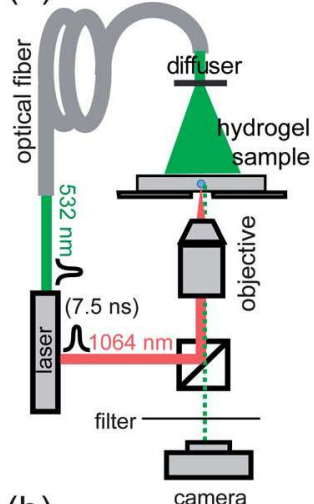

(b)
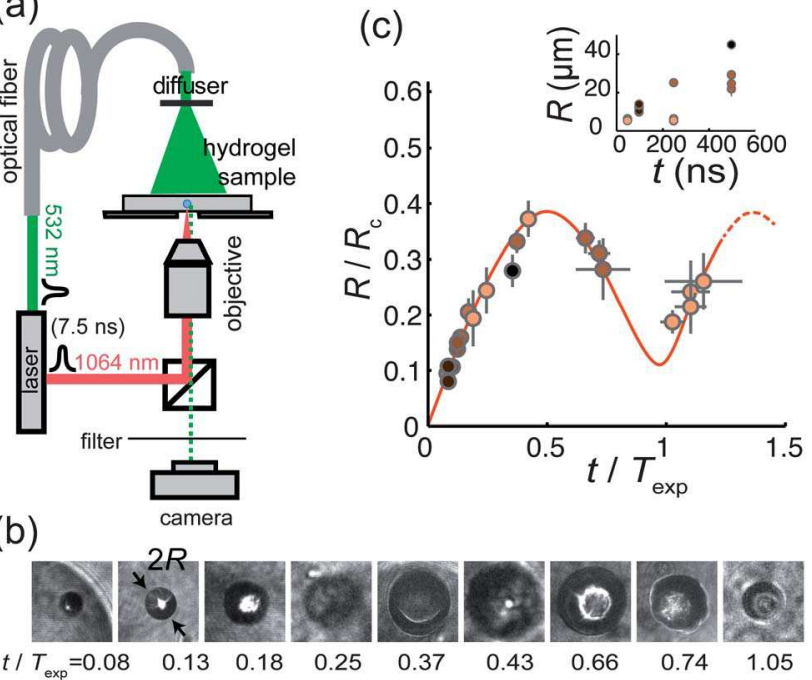

Fig. 3 Bubble dynamics measured by strobe photography (a) Experimental setup. The $1064 \mathrm{~nm}$ laser pulse triggers cavitation at $t=0$ in the metastable liquid, while the $532 \mathrm{~nm}$ pulse provides a short illumination at a time $t$ fixed by the optical fiber length. (b) Strobe photography for different time delays, the time $t$ is scaled by $T_{\exp }=1 / f$, where $f$ is given by eqn (1). (c) Radius of the bubble, scaled by the microcavity size, as a function of the scaled time after cavitation. The symbols are experimental measurements for different cavity sizes between 26 and $160 \mu \mathrm{m}$ (one cavitation event per point, the symbol color is lighter for small sizes). The line is a guide to the eye. Inset: raw data, before scaling.

period as the one detected by the photodiode (Fig. 2). The same scaling was used to reconstruct the image sequence of Fig. $3 \mathrm{~b}$, allowing direct visualization of the bubble oscillation.

Our strobe photography study thus demonstrates that the oscillations in Fig. 2 indeed arise from volume vibrations. In order to understand their unexpectedly high frequency and their dependence on $1 / R_{\mathrm{c}}$, we developed a simple model.

\section{Volume oscillations: model}

In a free liquid, bubble oscillations can be accommodated by displacing the liquid at long distances. However, in a fully confined environment, a bubble can grow only by compressing the liquid around it (at constant mass, neglecting evaporation) or by expanding the confining solid. This suggests that liquid compressibility (characterized by the bulk modulus $K_{\ell}$ ) plays a major role in the dynamics, as well as the elasticity of the solid material. We characterize the latter by an effective modulus $K_{\mathrm{c}}=$ $V_{\mathrm{c}} \mathrm{d} p / \mathrm{d} V_{\mathrm{c}}$, where $p$ is the liquid pressure and $V_{\mathrm{c}}$ is the volume of the microcavity. This modulus can be shown ${ }^{23,24}$ to be $K_{\mathrm{c}}=4 G / 3$ for a spherical geometry, $G$ being the shear modulus of the solid.

We describe the bubble as a dynamical system with only one degree of freedom: its radius $R$. For simplicity, we assume that the bubble is centered in the middle of the cavity, which allows us to use spherical coordinates.

Variations of the liquid pressure $\mathrm{d} p$ are associated with changes of the bubble volume $\mathrm{d} V$ as follows, $\mathrm{d} V=\mathrm{d} p / K_{\ell} \times V_{\ell, 0}+$

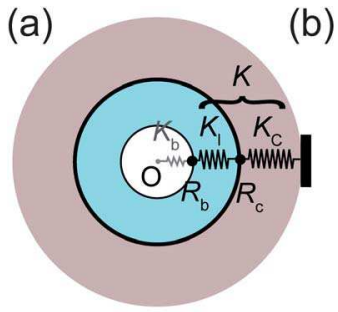

(b)

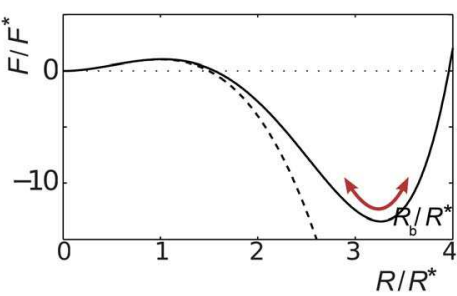

Fig. 4 (a) Sketch of the system, using mechanical analogues with springs for the bubble (white), liquid (blue) and solid (brown) elasticities. (b)Theoretical free energy potential $F$ to nucleate a bubble of radius $R$, with a potential well explaining the stable equilibrium radius $R_{\mathrm{b}}$ of the bubble and the origin of the oscillations. Around $R_{\mathrm{b}}$ the system is harmonic and can be described as the assembly of springs pictured on panel (a). The dashed line stands for the case of a bubble in an infinite liquid (classical nucleation theory), the solid line for a bubble in a confined environment. The radius is scaled by the critical radius $R^{*}$ $=2 \sigma /\left(-p_{0}\right)$ and the potential is scaled by the height of the nucleation barrier $F^{*}=\frac{4}{3} \pi R^{* 2} \sigma$. Here the confinement is chosen to be very strong to enhance this barrier.

$\mathrm{d} p / K_{\mathrm{c}} \times V_{\mathrm{c}, 0}$ where $V_{\ell, 0}$ and $V_{\mathrm{c}, 0}$ are reference volumes of respectively the liquid and the cavity. Taking the liquid before cavitation as the reference state, for which $R=0$ and $p=p_{0}<0$, one has $V_{\ell, 0}=V_{\mathrm{c}, 0}=4 \pi R_{\mathrm{c}}{ }^{3} / 3$ and the variations of liquid pressure as a function of bubble size are

$$
p(R)=p_{0}+K\left(\frac{R}{R_{\mathrm{c}}}\right)^{3}
$$

where we defined the effective bulk modulus $K=1 /\left(K_{\ell}{ }^{-1}+K_{\mathrm{c}}{ }^{-1}\right)$ that takes into account the compressibilities of both the liquid and the solid confinement (see Fig. 4a). Note that this expression is equivalent to the formula for springs in series.

This allows us to modify the bubble free energy from classical nucleation theory, ${ }^{25,26}$ which assumes that $p$ is independant of the bubble size, by incorporating compressibility effects. We also include the possibility of having $N$ particles of a noncondensable gas inside the bubble, to allow a comparison with Minnaert's case of air bubbles in water. This gives the following expression from the free energy: $₫$

$$
F=4 \pi R^{2} \sigma+\frac{4 \pi}{3} R^{3} p_{0}+\frac{2 \pi}{3} K \frac{R^{6}}{R_{\mathrm{c}}{ }^{3}}-3 N k_{\mathrm{B}} T \ln (R) .
$$

The first and last terms on the right hand side represent respectively the contributions of surface tension $\sigma$ and of the non-condensable gas, where $k_{\mathrm{B}}$ is the Boltzmann constant and $T$ is the temperature (we consider the isothermal case for the sake of simplicity). The two other terms were obtained by calculating the work of pressure forces $\int p \mathrm{~d} V$. We did not explicitly include the vapor pressure $p_{\text {sat }}$ of the liquid in the model. However our

I The notation $\ln (R)$ actually means $\ln \left(R / R_{\text {ref }}\right)$ where $R_{\text {ref }}$ is a reference radius that can be arbitrarily chosen as it just shifts the free energy by a constant amount and thus does not change the physical results. Please also note that all our calculations assume that the volume of the bubble remains small compared to the cavity size, which is justified in our experiments by the fact that $\left(R_{\mathrm{b}} / R_{\mathrm{c}}\right)^{3}=\beta^{3} \ll 1$, since $\beta^{3} \simeq$ 0.03 . This allows us to use linear elasticity approximations. 
expressions are still valid if $p$ does not represent the actual liquid pressure $p_{\ell}$, but $p=p_{\ell}-p_{\text {sat }}$. In most practical situations, including our experiments, $p_{\text {sat }}$ is negligible compared to the other pressures involved, and using $p$ as the liquid pressure is a very good approximation.

A typical shape of the free energy is shown in Fig. 4b. It exhibits a potential well corresponding to the equilibrium bubble position $R_{\mathrm{b}}$, around which oscillations may occur. The equilibrium occurs at a value $R_{\mathrm{b}}$ that is given by $(\mathrm{d} F / \mathrm{d} R)_{R=R_{\mathrm{b}}}=0$. The equilibrium condition can be written as

$$
p_{0}+K\left(R_{\mathrm{b}} / R_{\mathrm{c}}\right)^{3}=p_{\mathrm{g}, \mathrm{eq}}-2 \sigma / R_{\mathrm{b}}
$$

where $p_{\mathrm{g} \text {,eq }}=N k_{\mathrm{B}} T /\left(4 \pi R_{\mathrm{b}}{ }^{3} / 3\right)$ is the equilibrium gas pressure, and $p_{0}+K\left(R_{\mathrm{b}} / R_{\mathrm{c}}\right)^{3}$ can be recognized as the equilibrium liquid pressure $p_{\text {eq }}=p\left(R_{\mathrm{b}}\right)$ from eqn (2). This shows that the equilibrium radius is a function of the initial negative pressure $p_{0}$.

For small deviations around $R_{\mathrm{b}}, F(R)$ is quadratic and the system can be described as a harmonic oscillator of stiffness $k=$ $\left(\mathrm{d}^{2} F / \mathrm{d} R^{2}\right)_{R=R_{\mathrm{b}}}$. Inertia comes from the liquid that is set into motion during the bubble oscillation. In an infinite liquid, for radially oscillating bubbles, this can be taken into account ${ }^{27}$ by using an effective mass $m_{\text {eff }}=4 \pi \rho R_{\mathrm{b}}{ }^{3}$, the kinetic energy being $E_{\mathrm{k}}=\frac{1}{2} m_{\mathrm{eff}} \dot{R}^{2}$. We assume here that this formula remains valid in confined spaces, which we expect to be true as long as the bubble volume remains small compared to the total liquid volume. The natural oscillation frequency is then given by $f=$ $\left(k / m_{\mathrm{eff}}\right)^{1 / 2} / 2 \pi$, resulting in

$$
f=\frac{1}{2 \pi R_{\mathrm{b}}}\left(\frac{3}{\rho}\right)^{1 / 2} \sqrt{K\left(\frac{R_{\mathrm{b}}}{R_{\mathrm{c}}}\right)^{3}+K_{\mathrm{b}}},
$$

where $\rho$ is the density of the liquid at rest, and $K_{\mathrm{b}}=p_{\text {eq }}+4 \sigma / 3 R_{\mathrm{b}}$ $=p_{\text {g,eq }}-2 \sigma / 3 R_{\mathrm{b}}$ can be seen as the bulk modulus of the bubble in a free liquid, which includes contribution from both gas compressibility and surface tension. The oscillation frequency is thus set by different stiffness values that are sketched as springs in Fig. 4a.

Note that the initial negative pressure $p_{0}$ has been eliminated from the expression by using the equilibrium condition (4). In fact, eqn (5) is valid for any bubble oscillating around the radius $R_{\mathrm{b}}$ in a confined liquid: the bubble does not need to originate from a nucleation event in a liquid at negative pressure.

When $R_{\mathrm{c}} \rightarrow \infty$, the first term under the square root becomes negligible and the usual frequency of bubble oscillations in extended liquids ${ }^{3,5}$ is recovered.

In contrast, the first term is dominant when $R_{\mathrm{c}} / R_{\mathrm{b}} \ll\left|K / K_{\mathrm{b}}\right|^{1 / 3}$, i.e. when confinement and compressibility effects dominate surface tension and gas compressibility. We will now focus on the predictions in this confined regime. In this case, the right-hand side terms in eqn (4) are negligible, and the equilibrium radius of the bubble verifies the simple relationship $\beta=R_{\mathrm{b}} / R_{\mathrm{c}} \simeq\left(-p_{0} / K\right)^{1 / 3}$,

\| According to our definition, $p_{0}$ is the liquid pressure when $R=0$. It is equal to the liquid pressure before cavitation in the absence of non-condensable gas $(N=$ 0 ). If $N \neq 0$, the radius before cavitation is small but nonzero (corresponding to gas nuclei), and the pressure slightly differs from $p_{0}$. which allows us to rewrite the oscillation frequency (eqn (5)) in terms of the initial pressure $p_{0}$ and confinement radius $R_{\mathrm{c}}$, giving

$$
f \simeq \frac{1}{2 \pi R_{\mathrm{c}}}\left(\frac{3 K}{\rho}\right)^{1 / 2}\left(\frac{-p_{0}}{K}\right)^{1 / 6},
$$

which, noticeably, depends very weakly on the liquid pressure before cavitation.**

In our experiments, the elasticity of the spherical confinement material is ${ }^{28} K_{\mathrm{c}}=4 G / 3 \simeq 1 \mathrm{GPa}$. From the bulk modulus of water $K_{\ell} \simeq 2.2 \mathrm{GPa}$, we deduce the effective bulk modulus $K$ $\simeq 0.7 \mathrm{GPa}$. As the filling of the bubble by air occurs on timescales much longer than the bubble oscillatory dynamics, ${ }^{22}$ we will assume that there is no gas in the bubble during the oscillations $\left(p_{\mathrm{g} \text {,eq }}=0\right)$. And since the smallest bubble considered here had a radius of $R_{\mathrm{b}, \text { min }} \simeq 5 \mu \mathrm{m}$ one has $\left|K_{\mathrm{b}}\right|<2 \sigma /$ $3 R_{\mathrm{b}, \text { min }} \simeq 9 \mathrm{kPa}$ with $\sigma \simeq 0.07 \mathrm{~N} \mathrm{~m}^{-1}$ the surface tension of water. Then $\left|K / K_{\mathrm{b}}\right|^{1 / 3}>42$ while $R_{\mathrm{c}} / R_{\mathrm{b}} \simeq 3$. The previous condition for confinement is thus verified.

We thus predict $f \times R_{\mathrm{c}}=\mathscr{C}_{\mathrm{th}}=126 \mathrm{~m} \mathrm{~s}^{-1}$ from eqn (6) (using $\rho=10^{3} \mathrm{~kg} \mathrm{~m}^{-3}, p_{0} \simeq-20 \mathrm{MPa}$ and $\left.K=0.7 \mathrm{GPa}\right)$, in very good agreement with experimental results $\left(\mathscr{C}=120 \mathrm{~m} \mathrm{~s}^{-1}\right)$ despite the simplifying assumptions of our model. We also successfully predict the experimentally measured value of $\beta \simeq\left(-p_{0} / K\right)^{1 / 3} \simeq$ 0.3 .

Note that for an infinitely stiff confinement $\left(K_{\mathrm{c}} \rightarrow \infty\right)$, one has $K=K_{\ell}$. The stiffness of the system setting the oscillation frequency is then purely the compressibility of the liquid. This helps to understand why oscillations are so fast in fully confined environments, as liquids (and solids) are much less compressible than gases and thus "stiffer".

An interesting consequence of the high frequency of these oscillations is that acoustic radiation is emphasized (with radiated power ${ }^{27}$ proportional to frequency at a power 4 ), which may explain the fast damping of the measured oscillations. Vibrations of bubbles in confined environments should thus be associated with short but intense acoustic signals.

\section{Shape-related dynamics: ejection and fragmentation}

In addition to high-frequency volume oscillations, we observed other unusual phenomena associated with spontaneous cavitation in a confined liquid at negative pressure.

First, in the biggest microcavities that we studied, several bubbles are visible in the pictures after cavitation, usually with one bubble much larger than the other ones (Fig. 5a). One may wonder if these multiple bubbles arise from several nucleation events in the microcavity, or originate from the breakup of a single initial bubble.

Second, the bubble is consistently seen moving away from the walls after cavitation (i.e. towards the center of the

** Note that in the case of a heterogeneous nucleation process, the surface terms would be modified in eqn (3), and in the expression of $K_{\mathrm{b}}$. However, under the present conditions of strong confinement, the effect would be negligible because of the predominance of the compressibility term. 

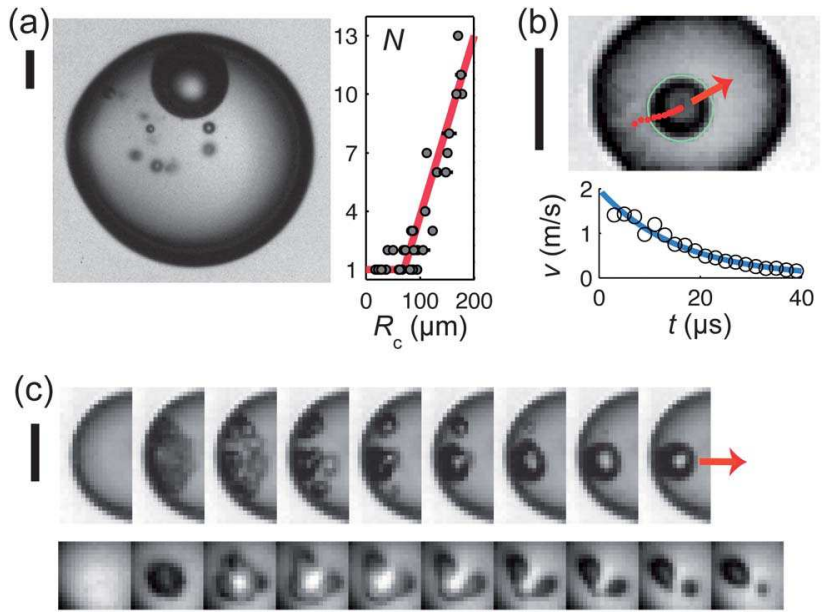

Fig. 5 Video recordings of the cavitation process at different timescales (decreasing from a to c). (a) Image taken from a horizontal viewing axis $200 \mathrm{~ms}$ after cavitation. The biggest bubble has risen to the top of the microcavity due to buoyancy. It is accompanied by smaller bubbles, which rise slower. The number $N$ of visible bubbles after cavitation is plotted as a function of cavity radius, the red line being a guide for the eye. (b) At shorter timescales $(\sim 10 \mu \mathrm{s})$, bubbles are seen moving away from the walls of the microcavity at speeds in the range 1-10 $\mathrm{m} \mathrm{s}^{-1}$. The red dots are successive positions of the bubble center after cavitation, and the arrow indicates the direction of motion. The bubble speed (circles: measured/line : exponential fit) decreases with time owing to viscous dissipation and the ejection motion ends typically close to the center of the microcavity. (c) Even shorter timescales: two high-speed cavitation sequences at 500000 frames per second ( $2 \mu$ s between successive images) for microcavities of radius $R_{\mathrm{c}} \simeq 60 \mu \mathrm{m}$. Top: cavitation starts on the wall in the left part of the microcavity. The red arrow symbolizes the ejection motion of the bubble described in panel b. Bottom: similar sequence but seen through the cavity wall: it is equivalent to the other sequence, but seen at a 90 degree angle. All scale bars: $50 \mu \mathrm{m}$.

microcavity in our spherical geometry), at speeds of the order of several meters per second (Fig. 5b). This ejection is surprising because in extended liquids, cavitation bubbles are usually seen to move towards solid boundaries ${ }^{2}$, pushed by liquid jets that develop during bubble oscillations.

In order to understand these remarkable features, we recorded high-speed camera movies (500 000 frames per second) of the cavitation process, which help to clarify the evolution of the bubble shape (Fig. $5 \mathrm{c}$ ).

From the recordings we notice that the initial position of the bubble in the movies is always against the wall of the microcavity. This suggests that nucleation occur on the wall, presumably due to a heterogeneous cavitation process. Please note that the second images of the sequences in Fig. $5 \mathrm{c}$ are blurred. This is due to the fact that the fast volume oscillation dynamics that we described previously happens immediately after cavitation, at timescales too short to be resolved by the high-speed camera, resulting in motion-blur. As a result, the following shape dynamics captured by the high-speed camera occurs after damping of the initial volume oscillations.

Our recordings also show that the initial bubble becomes toroidal, as shown in the third image of each sequence in Fig. 5c. This phenomenon is known to happen in cavitation dynamics close to walls, due to the focusing of jets through the bubble and towards the solid surface during bubble collapse.,29 But while in a free liquid the bubble would tend to completely collapse after a few oscillations, here the bubble oscillates around a non-zero volume. This entails the development of a much richer dynamics, as the toroidal bubble destabilizes quickly. Fragmentation is likely to be the consequence of the Rayleigh-Plateau instability of the tubular (toroidal) shape of the bubble, driven by the surface tension $\sigma$. Indeed, the characteristic capillary time $\tau_{\sigma}=\sqrt{\rho R_{\mathrm{b}}{ }^{3} / \sigma}$ is of the order of microseconds for $R_{\mathrm{b}} \sim 10 \mu \mathrm{m}$, in agreement with the sequences in Fig. 5c. This phenomenon eventually leads to bubble breakup, explaining the multiple bubbles in Fig. 5a. We hypothesize that this phenomenon is mostly seen in bigger microcavities because inertia is stronger compared to surface tension, making jets more likely to pierce the bubble. $\dagger \dagger$

Noticeably, a ripening process, which might be similar to Ostwald ripening, made some of the fragmented bubbles recombine, leading to a bubble that can be single or accompanied by much smaller sister bubbles.

After formation the biggest bubble is spread along the wall. The bubble shape, being that of a compressed sphere against the wall, evolves back to a spherical shape over time. This consideration leads us to propose the following explanation for bubble expulsion: as with a balloon flattened on the ground which is suddenly released, the return to a spherical shape is able to entail propulsion away from the wall. The driving force for the motion is membrane tension for the balloon and surface tension $\sigma$ for the bubble. The ejection speed can be evaluated by balancing the surface energy $E_{\mathrm{s}} \sim \sigma R_{\mathrm{b}}{ }^{2}$ and the translation kinetic energy $E_{\mathrm{t}} \sim \rho R_{\mathrm{b}}{ }^{3} \mathrm{v}^{2}$, leading to

$$
v \sim \sqrt{\frac{\sigma}{\rho R_{\mathrm{b}}}} .
$$

This predicts velocities of the order of $2 \mathrm{~m} \mathrm{~s}^{-1}$ for bubbles of radius $15 \mu \mathrm{m}$ such as the one in Fig. 5b. This prediction is thus in very good agreement with the observed velocities, and also explains the tendency of ejection velocities to decrease when the bubble size increases (data not shown here). Our experimental data are thus compatible with the mechanism of capillary ejection, very different from the jet-propelled bubbles seen in cavitation close to walls in extended liquids.

Such a fast auto-ejection from the solid surface propels bubbles towards the liquid. It seems important to note that, as a result, observations by eye or with a camera at a too low frame rate ( $<100000$ fps typically) would not resolve this motion and might lead to the erroneous conclusion of homogeneous nucleation in the bulk of the liquid.

In summary, fast inertial volume oscillations close to the wall lead to the development of violent jets that make the bubble toroidal. This shape is unstable and bubble fragmentation occurs. Ripening of these fragments produces a big

$\dagger$ The Weber number, which compares inertia with surface tension, can be expressed as We $=\rho \mathscr{C}^{2} R_{\mathrm{b}} / \sigma$ in our system. Since $\mathscr{C}$ is a constant and $R_{\mathrm{b}}=\beta \times$ $R_{\mathrm{c}}$, the Weber number increases linearly with $R_{\mathrm{c}}$. Its value is of the order of $10^{3}$ for the smallest bubbles that we studied. 
bubble and possibly smaller bubbles. The big bubble is then quickly ejected away from the wall, due to capillary phenomena, before rising to the top of the microcavity due to buoyancy.

Remarkably, our model of volume oscillations, based on a spherical bubble assumption, still describes accurately the experimental results in spite of this highly non-spherical dynamics. Admittedly, the fragmentation dynamics is not expected to influence volume oscillations as the former occurs on much longer timescales than the latter. But jetting and toroidal bubble formation happen because of the oscillations and thus probably simultaneously with them.

We also remark that while both shockwaves and jets are usually observed in un-confined bubble dynamics, here we never observed any sign of a shock front in the images obtained by strobe photography. Shock wave emission usually appears at the last stage of the bubble collapse when the bubble decelerates rapidly. Strong acoustic driving e.g. in single bubble sonoluminescence, and for largely empty vapor bubbles has demonstrated this shock wave emission. In contrast in this experiment the bubbles oscillate around a comparably large volume, i.e. the equilibrium radius. Thus we expect that the bubble collapse is generally weaker in our system.

\section{Conclusion and perspectives}

To conclude, we have explored a new class of bubble dynamics, occurring in a liquid under static tension that is fully confined in an elastic solid.

Static tension implies in particular that both the liquid volume and the confinement size relax after nucleation so that the bubble does not eventually collapse. This different outcome enables the development of interesting hydrodynamic phenomena, such as the formation, fragmentation and ripening of a toroidal bubble or its capillary ejection away from the wall that are very different to what can be seen for free cavitation bubbles. Note that the absence of complete bubble collapse probably means that the shock-waves usually associated with cavitation events do not develop here, but this does not necessarily mean that confined cavitation is a gentle process. The formation of a toroidal bubble indeed suggests the occurrence of strong liquid jets and thus the possibility of erosion effects.

Confinement implies that liquid and solid compressibilities (rather than the gas compressibility in free liquids) play a major role in the bubble vibratory dynamics, making volume oscillations extremely fast. This is an interesting example where the compressibility of a liquid may play a dominant role in the fluid mechanics of a system, and is a good illustration of a "visible" effect of this compressibility, often assumed to be zero. We proposed a geometrical criterion indicating when this compressibility effect dominates in the bubble dynamics. We showed that the bubble oscillation frequency was in the $\mathrm{MHz}$ range and mostly depends on the confinement size, with only a weak dependence on the (negative) liquid pressure before cavitation.

This particular oscillation signature should be detectable in acoustic signals linked to bubble oscillations, which opens the perspectives to get information on the geometry of the confinement by acoustical means. Possible applications of such a non-invasive technique range from cavitation monitoring in porous media, quartz inclusions or soil tensiometers, to the pressing problem of understanding cavitation in plants. Indeed, acoustic emissions are used to estimate the vulnerability of trees to cavitation during drought periods, which are expected to be more frequent in the future. ${ }^{30}$ Note that our predictions for the natural volume oscillations are valid for any confined bubble, whether it arises from a cavitation event or not, and may thus be applied to bubbles permanently existing in a confined liquid, and excited by an external source such as an acoustic field.

\section{Acknowledgements}

The authors acknowledge financial support from the FrenchSingapore Merlion Grant no. 2.08.09.

\section{References}

1 A. Prosperetti, L. A. Crum and H. C. Pumphrey, J. Geophys. Res., 1989, 94, 3255-3259.

2 W. Lauterborn and T. Kurz, Rep. Prog. Phys., 2010, 73, 106501.

3 C. E. Brennen, Cavitation and Bubble Dynamics, Oxford University Press, Oxford, 1995.

$4 \mathrm{H}$. Becher and P. Burns, Handbook of contrast echocardiography, Springer Verlag, 2000.

5 M. Minnaert, Philos. Mag., 1933, 16, 104.

6 A. Prosperetti, Phys. Fluids, 1987, 30, 3626-3628.

7 J. R. Blake and D. C. Gibson, Annu. Rev. Fluid Mech., 1987, 19, 99-123.

8 M. Strasberg, J. Acoust. Soc. Am., 1953, 25, 536-537.

9 C. W. M. Van Der Geld and J. G. M. Kuerten, J. Fluid Mech., 2009, 640, 265-303.

10 H. N. Oguz and A. Prosperetti, J. Acoust. Soc. Am., 1998, 103, 3301-3308.

11 S. Martynov, E. Stride and N. Saffari, J. Acoust. Soc. Am., 2009, 126, 2963-2972.

12 Q. Zheng, D. J. Durben, G. H. Wolf and C. A. Angell, Science, 1991, 254, 829-832.

13 M. E. M. Azouzi, C. Ramboz, J.-F. Lenain and F. Caupin, Nat. Phys., 2013, 9, 38-41.

14 M. T. Tyree and J. S. Sperry, Annu. Rev. Plant Physiol. Plant Mol. Biol., 1989, 40, 19-36.

15 H. Cochard, C. R. Phys., 2006, 7, 1018-1026.

16 K. T. Ritman and J. A. Milburn, J. Exp. Bot., 1990, 41, 11571160.

17 G. W. Scherer and D. M. Smith, J. Non-Cryst. Solids, 1995, 189, 197-211.

18 D. Or and M. Tuller, Water Resour. Res., 2002, 38, 19.

19 M. T. Tyree and M. A. Dixon, Plant Physiol., 1983, 72, 10941099.

20 X. Noblin, N. O. Rojas, J. Westbrook, C. Llorens, M. Argentina and J. Dumais, Science, 2012, 335, 1322.

21 T. D. Wheeler and A. D. Stroock, Nature, 2008, 455, 208-212. 
22 O. Vincent, P. Marmottant, P. A. Quinto-Su and C.-D. Ohl, Phys. Rev. Lett., 2012, 108, 184502.

23 L. D. Landau and E. M. Lifshitz, Theory of elasticity, Pergamon Press, 1970.

24 V. N. Alekseev and S. A. Rybak, Acoust. Phys., 1999, 45, 535540.

25 P. G. Debenedetti, Metastable Liquids: Concepts and Principles, Princeton University Press, 1996.

26 F. Caupin and E. Herbert, C. R. Phys., 2006, 7, 1000-1017.

27 T. G. Leighton, The acoustic bubble, Academic Press, 1994.
28 O. Vincent, PhD thesis, Univ. Joseph Fourier, Grenoble, France, 2012.

29 T. B. Benjamin and A. T. Ellis, Phil. Trans. Roy. Soc. Lond. Math. Phys. Sci., 1966, 260, 221-240.

30 B. Choat, S. Jans, en, T. J. Brodribb, H. Cochard, S. Delzon, R. Bhaskar, S. J. Bucci, T. S. Feild, S. M. Gleason, U. G. Hacke, A. L. Jacobsen, F. Lens, H. Maherali, J. MartinezVilalta, S. Mayr, M. Mencuccini, P. J. Mitchell, A. Nardini, J. Pittermann, R. B. Pratt, J. S. Sperry, M. Westoby, I. J. Wright and A. E. Zanne, Nature, 2012, 491, 752-755. 